\title{
Emergency Ad-Hoc Networks by Using Drone Mounted Base Stations for a Disaster Scenario
}

\author{
Margot Deruyck, Jorg Wyckmans, Luc Martens, Wout Joseph \\ Ghent University - iMinds - WAVES, Dept. of Information Technology, \\ Technologiepark-Zwijnaarde 15, B-9052 Ghent, Belgium, margot.deruyck@intec.ugent.be
}

\begin{abstract}
In case of a large scale disaster, the wireless access network can become quickly saturated. This is of course undesirable because for this kind of situations we actually need a reliable wireless connectivity. In this study, the potential of mounting LTE femtocell base stations on drones to offer an alternative for the saturated existing wireless infrastructure is investigated. Our preliminary results show that this a very promising approach although a high amount of drones are needed to cover all users in the city center of Ghent, Belgium during a $1 \mathrm{~h}$ intervention. The number of drones can be significantly reduced (up to $64 \%$ ) by using a more advanced type of drone, by decreasing the user coverage requirement $(11 \%$ less drones when requiring $80 \%$ instead of $90 \%$ ) or by increasing the fly height of the drones (about 10\% less drones needed when increasing the fly height by $10 \mathrm{~m}$ ). This study shows that it is interesting to further investigate the use of drones to provide an emergency wireless access network.
\end{abstract}

\section{INTRODUCTION}

Today our wireless access networks are very reliable. Whenever we want, we can access the internet, send messages or make a phone call. This connectivity has become a natural thing. In this respect, it is very logical that in case of a major emergency, we want to get in touch with our loved ones to let them know we are ok. However, this might not be possible due the saturation of the network. In August 2011, the annual music festival Pukkelpop in Kiewit, Belgium, with 600000 attendees was hit by a severe storm. Although the storm only lasted for a couple of minutes it caused major damage by uprooting trees and the collapse of some tents resulting in 140 injuries and even 5 deaths. The news about the storm quickly reached the media, and the network was saturated by people trying to get in touch with each other. Although it was still possible to use social media, calling and sending text messages were no longer possible. More recently, in March 2016, Brussels got hit by a terror attack. The network was not only saturated in Brussels, but also in big parts of the country phone calls were no longer possible. Also during major traffic jams on the high ways, the network might be locally saturated by people letting others know they will be late.

One way to offer a solution for this problem is to mount base stations on unmanned aerial vehicles (UAVs) like hot air balloons or drones. From now on, we will use the term drones. This form of mobile base stations can be used for a variety of use cases like for example to provide a network connection to unreachable places [1]. Such a network is called a FANET (Flying Ad-Hoc Network) [2]. There are different ways to realize such FANETs. For example, there can be one coordinator base station on the ground or satellite and all base stations have to connect to it. One of the major disadvantages is that all base stations need to be in range of the coordinator in order to send their data. Another way is to equip only a subset of the base stations with the infrastructure to connect with the back haul network. All other base stations have to connect with these base stations to send their data to the underlying network.

In this paper, the potential of mounting of LTE (Long Term Evolution) base stations on drones to offer a solution for those scenarios where the existing infrastructure is overwhelmed is investigated. To this end, a deployment tool is developed. This tool does not only allow to calculate the required amount of drones, but also the most optimal locations for these drones in order to maximize the user coverage taking into account the specifications of the base station and the power usage of both the base station and the drone.

The outline of this paper is as follows. In the next section, the methodology is described. First, the considered drone and LTE base station is discussed in detail. Second, the algorithm of the deployment tool is discussed, and finally, the assumed scenario is proposed. Section III presents the results obtained with the deployment tool and in Section IV we summarize the most important conclusions.

\section{Methodology}

\section{A. Scenarios}

As mentioned above LTE femtocell base stations will be placed on the drones. The LTE technology was chosen over WiFi due to the uncontrolled nature of the unlicensed band in which WiFi operates, causing a dramatically poor throughput when a lot of users are competing for the same resources. This is problematic for the considered use case as our main target is to reconnect a large number of users in the same place. Furthermore, LTE is optimized to handle (slow) moving users whereas WiFi is not [3]. A femtocell base station was chosen because of its small size and limited power consumption (i.e., $12 \mathrm{~W}$ ) [4]. Table I shows the link budget parameters for the considered scenario.

Fig. 1 shows the considered suburban area of $6.85 \mathrm{~km}^{2}$ in Ghent, Belgium. A 3D model (in shape file) of the city center 
TABLE I

LINK BUDGET PARAMETERS FOR THE LTE FEMTOCELL BASE STATION.

\begin{tabular}{|l|c|}
\hline Parameter & Value \\
\hline Frequency & $2.6 \mathrm{GHz}$ \\
\hline Maximum input power antenna & $33 \mathrm{dBm}$ \\
\hline Antenna gain base station & $4 \mathrm{dBi}$ \\
\hline Soft handover gain & $0 \mathrm{~dB}$ \\
\hline Feeder loss base station & $0 \mathrm{~dB}$ \\
\hline Fade margin & $10 \mathrm{~dB}$ \\
\hline Interference margin & $2 \mathrm{~dB}$ \\
\hline Receiver Signal-to-Noise Ratio (SNR) & $1 / 3 \mathrm{QPSK}=-1.5 \mathrm{~dB}$ \\
& $1 / 2 \mathrm{QPSK}=3 \mathrm{~dB}$ \\
& $2 / 3 \mathrm{QPSK}=10.5 \mathrm{~dB}$ \\
& $1 / 216-\mathrm{QAM}=14 \mathrm{~dB}$ \\
& $2 / 3 \mathrm{16}-\mathrm{QAM}=19 \mathrm{~dB}$ \\
& $1 / 264-\mathrm{QAM}=23 \mathrm{~dB}$ \\
& $2 / 3 \mathrm{64}-\mathrm{QAM}=29.4 \mathrm{~dB}$ \\
\hline Number of used subcarriers & 301 \\
\hline Total number of subscarriers & 512 \\
\hline Bandwidth & $5 \mathrm{MHz}$ \\
\hline Noise figure mobile station & $0 \mathrm{~dB}$ \\
\hline Implementation loss mobile station & $12.3 \mathrm{~dB}$ \\
\hline Shadowing margin & $0 \mathrm{~dB}(1 \mathrm{x} 1 \mathrm{SISO})$ \\
\hline MIMO gain & \\
\hline & \\
\hline & \\
\hline
\end{tabular}

gives all information about buildings in the environment and their shape and height. Based on this information, it is possible to determine if a user is indoor or outdoor and whether or not he is in Line-of-Sight of a certain base station.

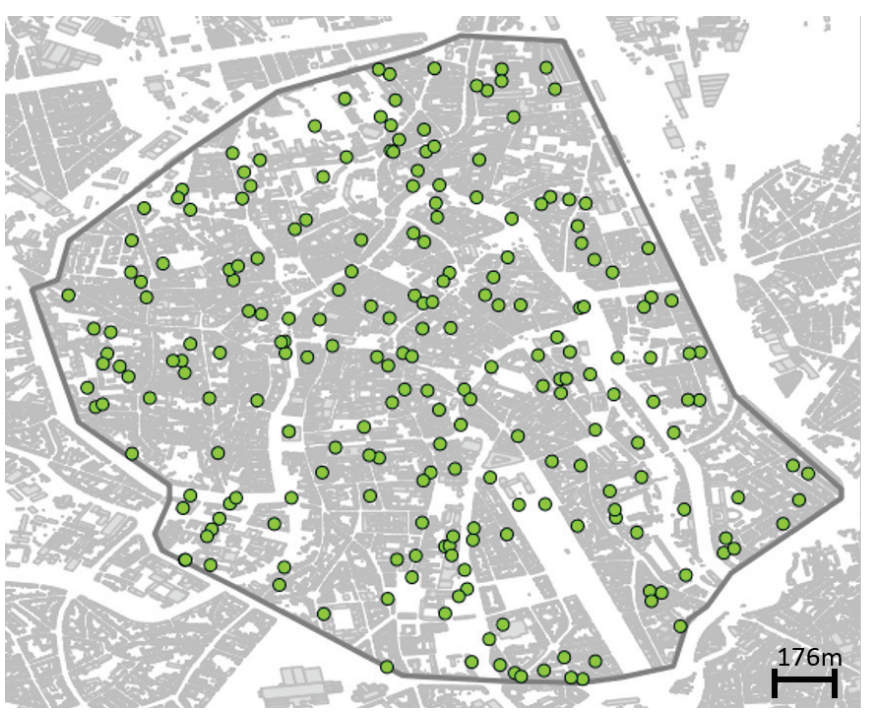

Fig. 1. The selected suburban area in Ghent, Belgium $\left(6.85 \mathrm{~km}^{2}\right)$. The green circles gives an example of the user distribution.

Table II describes the considered disaster scenario. We assume a worst case scenario in which the existing infrastructure of 75 base stations in the city center is unavailable due to e.g., a natural disaster. Drones will need to provide full coverage for the 224 users active in the area (worst case scenario, 5 p.m. as shown in [5] during $1 \mathrm{~h}$. The users are uniformly distributed over the area of interest as proposed in [5] and an example is shown in Fig. 1 (green circles). Analogously as the scenario in [5], some users request $64 \mathrm{kbps}$ to make a voice call, and others $1 \mathrm{Mbps}$ to make a data call (distributed according to [5]). Furthermore, a facility housing the drones equipped with base stations needs to be considered. This facility can be a kind of warehouse located in the city center or it can be a truck driving to the disaster site to minimize the flight time of the drones. In this scenario, a truck will bring all the drones to the city center, so the location of the facility storing all drones will be optimized to reduce fly time. The most optimal location for the facility is determined by using the mean value of the base stations' $\mathrm{x}$-coordinates, respectively $\mathrm{y}$-coordinates, as $\mathrm{x}$ coordinate and y-coordinate for the facility. Unless mentioned otherwise, the values of Table II are used for the different simulations. To predict the coverage and which users can connect to which drone mounted base station, the WalfishIkegami path loss model was considered [6] as we assume that the users will only connect to the base station when the drone is stabilized in the air. For the different results shown in the next section, 50 simulations were performed (because of the variability of the users' locations) and the $95^{\text {th }}$ percentile values are presented [5].

TABLE II

CONSIDERED DISASTER SCENARIO.

\begin{tabular}{|l|c|}
\hline Parameter & Assumption \\
\hline Number of users to reconnect & 224 (5 p.m.) \\
\hline Throughput demand & 1 Mbps (data) \& 64 kbps (voice) \\
\hline Location facility & Optimised \\
\hline Capacity facility & 300 drones \\
\hline Duration disaster/intervention & $1 \mathrm{~h}$ \\
\hline Fly height $h_{b}$ & $35 \mathrm{~m}$ to $h_{\max }$ \\
\hline Maximal fly height $h_{\max }$ & 0 \\
\hline $\begin{array}{l}\text { Base stations available } \\
\text { from fixed infrastructure }\end{array}$ & \\
\hline
\end{tabular}

\section{B. Drone mounted LTE femtocell base station}

As mentioned above, the LTE femtocell base stations are brought in place by using UAVs or drones. The base stations are mounted on drones which will fly them to the right location. Fig. 2 visualizes the process. As shown in Step 1 of Fig. 2, a facility is housing multiple drones equipped with base stations. From this facility, the necessary amount of drones are dispatched to the place of the disaster (Step 2). Upon arrival, the users can connect to the base stations mounted on the drones (Step 3). The drones can flight back to the facility (Step 4) when the disaster is over, when a permanent solution is provided or to recharge their battery.

Both helicopter drones and winged drones are qualified to mount base stations [7], [8], [9]. A helicopter drone has a similar design as helicopters, meaning it flies by using propellers [7], [8]. Winged drones have a similar design as an airplane, meaning they stay in the air using wings instead of propellers [9]. [7] and [8] show that it is feasible to mount the necessary equipment of a base station on a drone and have it located in a fixed place for a certain amount of time. However, when using a winged drone, a higher variability of the signal due to the constant movement of the drone is noticed [9]. This variability makes it a lot harder to predict the available signal strength. As a helicopter drone does not 


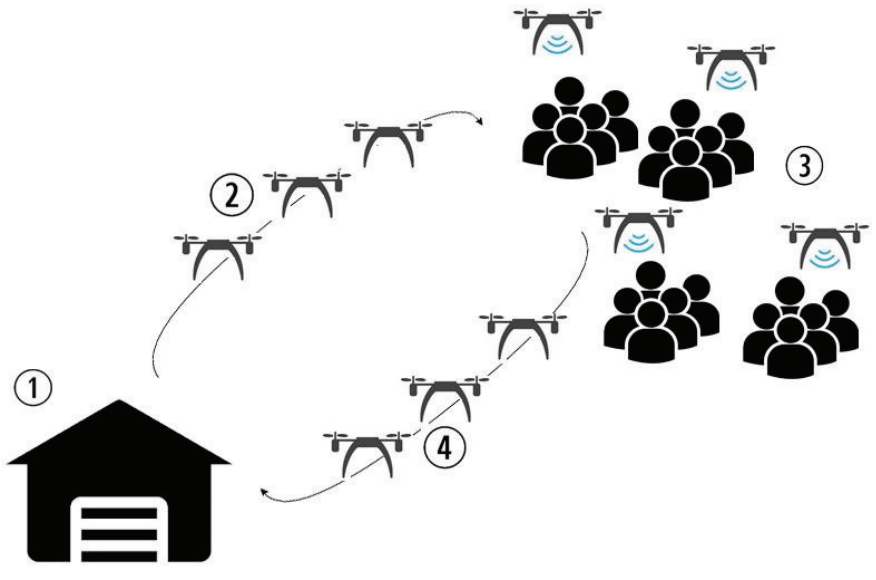

Fig. 2. Different steps to establish the drone network.

have this disadvantage, this type of drone is considered in this study. Two types of helicopter drones are used. The first one, here called 'Type 1', is an off the shelf drone which is affordable for the big public. More information about this drone can be found in [10]. The second one, from now on referred to as 'Type 2', is a more advanced one and thus also a lot more expensive. It can carry more weight and stay up in the air up to six times longer than the Type 1. An example of this type of drone is the microdrones md4-1000 model [11]. The most relevant parameters for this study (the speed of the drone, power usage, and battery) can be found in Table III for both types of drones.

TABLE III

SPECIFICATIONS OF THE DRONE CARRIER.

\begin{tabular}{|l|c|c|}
\hline Parameter & Type 1 & Type 2 \\
\hline Average carrier speed & $15.0 \mathrm{~m} / \mathrm{s}$ & $12.0 \mathrm{~m} / \mathrm{s}$ \\
\hline Carrier power usage & $5.0 \mathrm{~A}$ & $13.0 \mathrm{~A}$ \\
\hline Carrier power capacity & $2.0 \mathrm{Ah}$ & $17.33 \mathrm{Ah}$ \\
\hline Carrier battery voltage & $14.3 \mathrm{~V}$ & $22.2 \mathrm{~V}$ \\
\hline
\end{tabular}

Two other important parameters are the height $h_{b}$ between the drone and the surface above which it is hoovering and the maximal height $h_{\max }$ a drone can reach. This surface could be the street or the rooftop of a building in which a user is located. It is important to have this margin since it is undesirable to place a drone too close to the surface where people could damage it or too close to hanging cables and other obstacles around the area. Both parameters will have their influence on the results.

\section{Deployment tool}

The algorithm of the deployment tool consists of four different steps. First, the traffic is generated. We use the same approach as proposed in [5], which is based on real data from a mobile operator. Two categories of users are assumed as mentioned above: voice call users requiring $64 \mathrm{kbps}$ and data call users requiring $1 \mathrm{Mbps}$. The users are uniformly divided over the considered area, meaning that every location in the area has to same chance to be chosen as a location for the user. Second, a list of all possible base station locations is generated. The algorithm tries to place a drone above every user and marks this location as a possible base station location. Therefore, we need to know if the location will intersect with a building. If so, the algorithm will place the potential location above the building (building height + a certain margin) where the user is located in. If the user is outdoor, the potential location that will be added to the list is above the user at a height of $4 \mathrm{~m}$. The previous steps will be repeated until all user locations are investigated. If all user locations are considered, this part of the algorithm is finished and it will move to the next phase. Fig. 3 shows the different steps of the next phase, which is the creation of the actual network based on the list of possible base station locations.

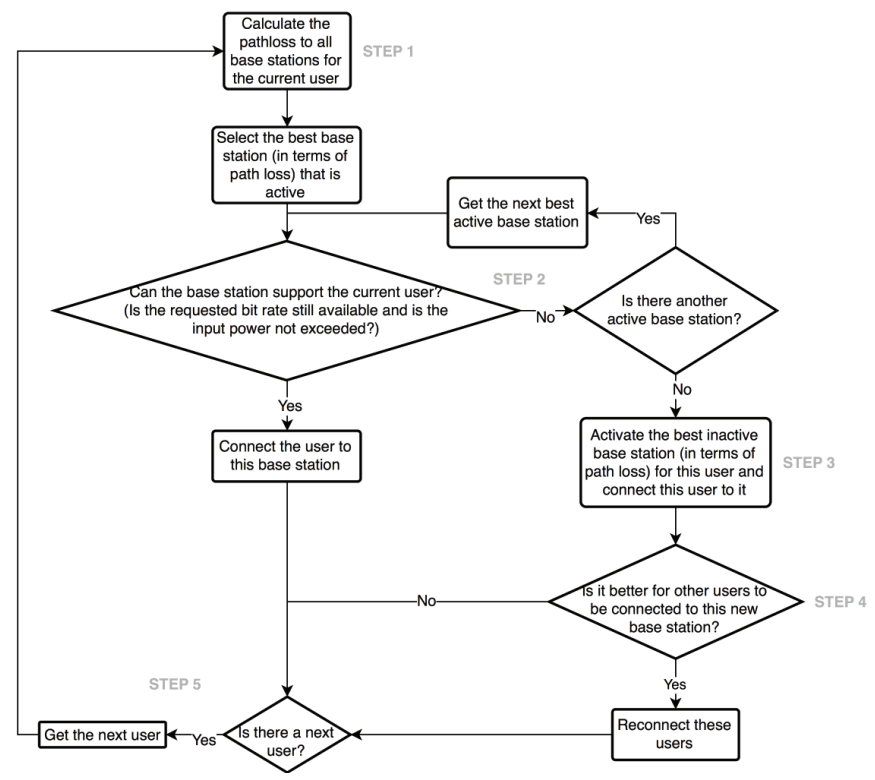

Fig. 3. Flow diagram of the network design based on the possible base station location list.

In order to determine which base station location is the most appropriate one to connect the user with, the path loss between the user and all possible base stations is calculated (Step 1). The user will be connected to the base station corresponding with the lowest path loss. This is of course only possible if the base station is already active and if the base station can still support the bit rate required by the considered user (Step 2). If the base station can no longer offer this bit rate, the algorithm checks the second best base station (i.e., the base station from which the user experiences the second lowest path loss) that is active and so on, until a feasible, active base station is found. In case no active base station can be found, the algorithm will activate the most appropriate base station from the inactive ones (Step 3) to connect the user with. In order to spread the load across the network, we check if it is possible to switch already connected users to this newly activated base station (Step 4). This can only be done when the user experiences a lower path loss from the newly activated base station than from the one 
he or she was connected to. The algorithm will repeat these previous steps until all users are assigned to a certain base station (Step 5). Note that multiple base stations and drones will be needed on one location to cover the users continuously through the whole intervention. The outcome of this phase will be a list of the most optimal base station locations. After this phase, we will know how many and which users are connected to each of the chosen base station locations. In order to create this list, no restrictions of the amount of drones (and thus base station locations) are considered yet. This will be done by the next phase of the algorithm. The information from this phase about the most optimal base station locations will be used to distinguish between the most important and less important base station locations.

Fig. 4 shows how the algorithm is dealing with the constraints of the number and specifications (e.g., flight time) of the drones.

The algorithm starts with sorting the list of optimal base station locations from the previous phase. There are two ways in which this list can be sorted (Step 1). The first way is according to the amount of users that is covered by the base station. The more users they cover, the higher the chance they will be provided by the drones. This approach will be called 'hot spot' from now on. The second way to sort the list is to use the amount of users in relation to the necessary amount of drones carrying the base stations. If a base station location connects a lot of users without requiring a lot of drones, its chance of being provisioned will increase. This approach will be called 'ratio' from now on, as it takes into account the ratio of required drones versus the number of connected users. Based on the list of sorted base stations, the algorithm is provisioning the different locations with the right amount of drones based on the available drone capacity. To do this, two parameters are accounted for: the flight time from the facility to the required location (Step 2) and the power usage of the drone and the base station (Step 3). Based on these two values, the time the battery will last can be calculated (Step 4). Once we know how long a drone and base station can be in the air, we can determine how many of drones are necessary to bridge the duration of the entire intervention (Step 5). If this amount is still available at the facility, the location is marked as feasible and the necessary amount of drones is reserved at the facility (Step 6). In case the round trip time alone drains already the battery, the location will be marked as infeasible. The algorithm stops when all locations are checked or when there are no more drones available at the facility.

\section{RESULTS}

In this section, simulations are performed by using the deployment tool of Section II-C for the scenario proposed in Section II-A.

\section{A. Capacity of the facility}

As mentioned above, the facility is a location where the drones are stored when they are not in use and from which

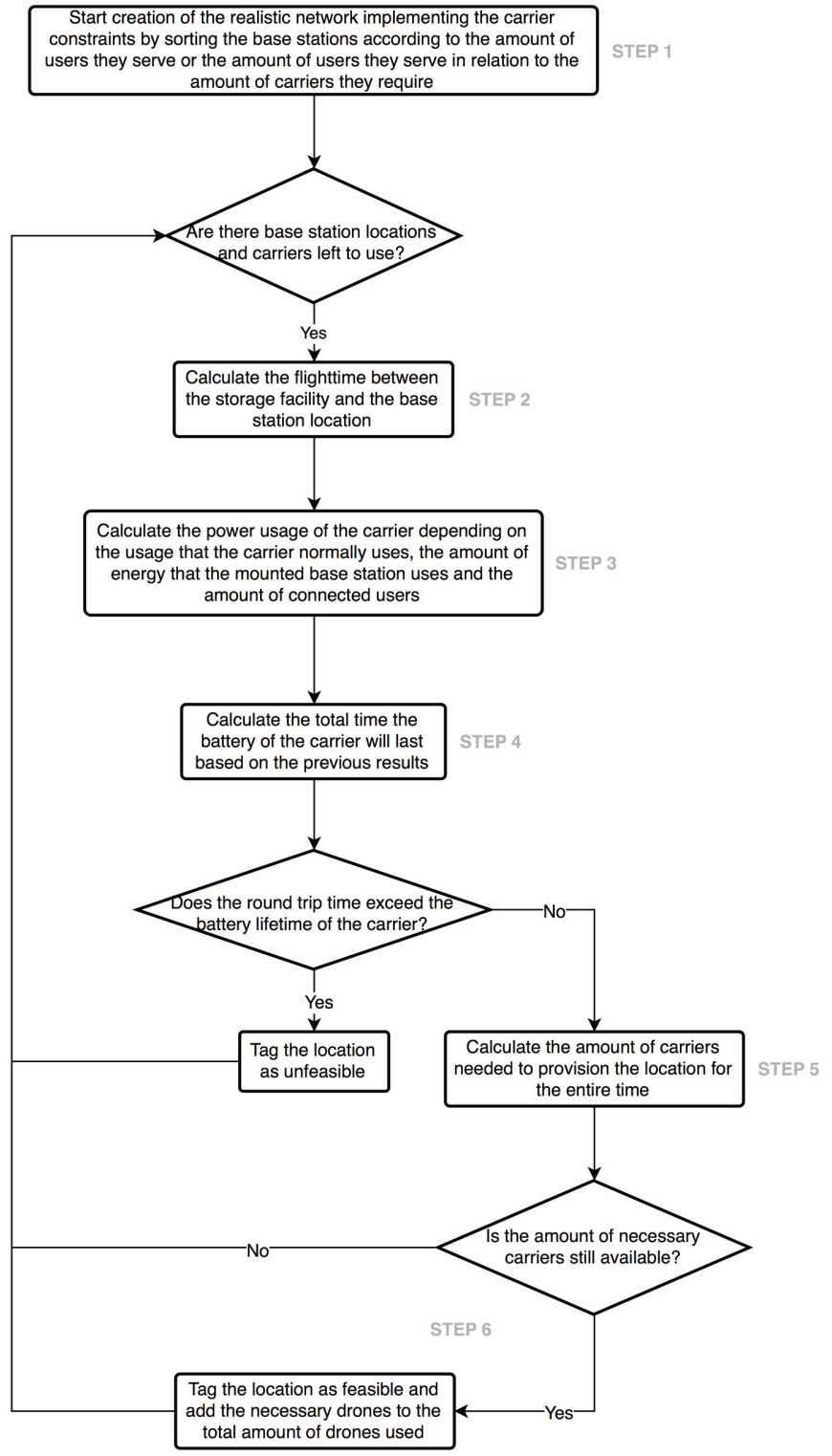

Fig. 4. Flow diagram of applying the restrictions to the designed network.

the drones will fly to the location they were assigned to. The capacity of a facility is the amount of drones that reside in such a facility and represents the number of available drones to cover the considered area. Fig. 5 shows the influence of the facility's capacity on the user coverage when using the ratio (in blue) and the hotspot approach (in orange).

Based on Fig. 5, we conclude that, for the considered scenario, 1100 Type 1 drones are needed to cover most of the users (i.e., $99.6 \%$ to $99.8 \%$ ) for one hour, while when using Type 2 drones only 400 are required. When less drones are available, a linear relation between the capacity of the facility and the user coverage is found for both types. An additional capacity of 100 drones leads to an increase of approximately $10 \%$ and $20 \%$ for the user coverage when using Type 1 and Type 2 drones, respectively. Comparing the two techniques, ratio and hotspot, the same amount of drones are needed, 


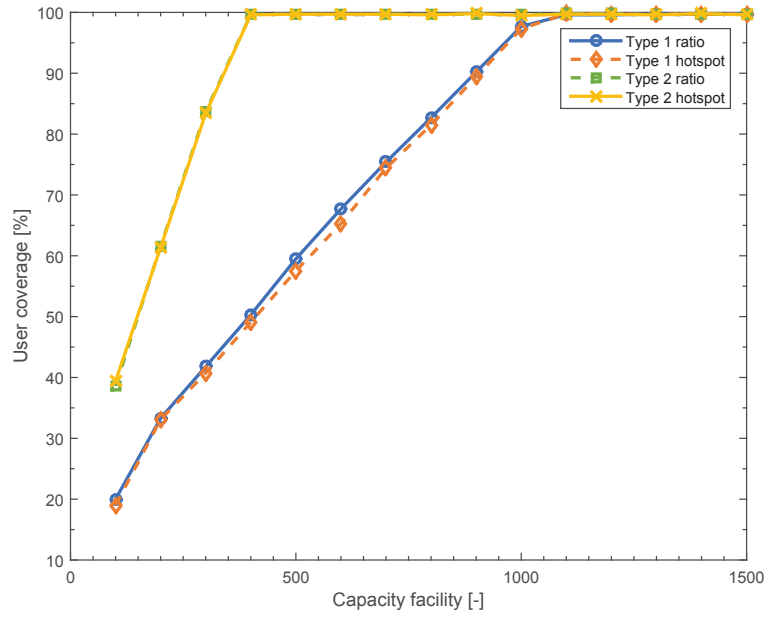

Fig. 5. Influence of the capacity of the facility on the user coverage.

however, the ratio approach performs slightly better than the hotspot technique. In the considered scenario, the difference between the two techniques is not significant (on average a $0.01 \%$ to $0.22 \%$ higher user coverage is obtained when using the ratio technique) due to the fact that the facility is optimally located and for both techniques the locations they provide first will be close. However, when the facility is not placed optimally, the difference between both techniques will become larger due to the smarter resource allocation method used by the ratio technique. From now on, the ratio technique will only be considered.

\section{B. Influence of the intervention duration}

In this section, the influence of the intervention duration on the results is investigated. The intervention duration is here defined as the interval between the moment that connecting to the existing infrastructure becomes impossible and the moment when a permanent solution is installed or when the existing infrastructure becomes available again. Fig. 6 shows how the user coverage is influenced by the intervention duration. This relation is clearly an exponential decrease: the longer the duration, the less coverage the drones can provide. As the facility capacity is fixed to 300 drones, it will no longer be possible to cover the most distant users due to the fact that the algorithm provides the locations that reconnect the most users with the lowest number of drones first (see Section II-C). The relation converges towards approximately 3.5\% when a duration of 20000 s i.e., approximately $5.5 \mathrm{~h}$, or higher is reached when using Type 1 drones. For the Type 2 drone, it converges to approximately $10 \%$ for a duration longer than $24 \mathrm{~h}$. When the intervention duration increases, more drones will be needed to continuously cover the same number of users. Because the drones that become available by not covering the most distant users will be used to continuously cover the users closest to the facility, the relation converges to approximately $3.5 \%$ and $10 \%$ for the Type 1 and 2 drones, respectively, instead of $0 \%$.

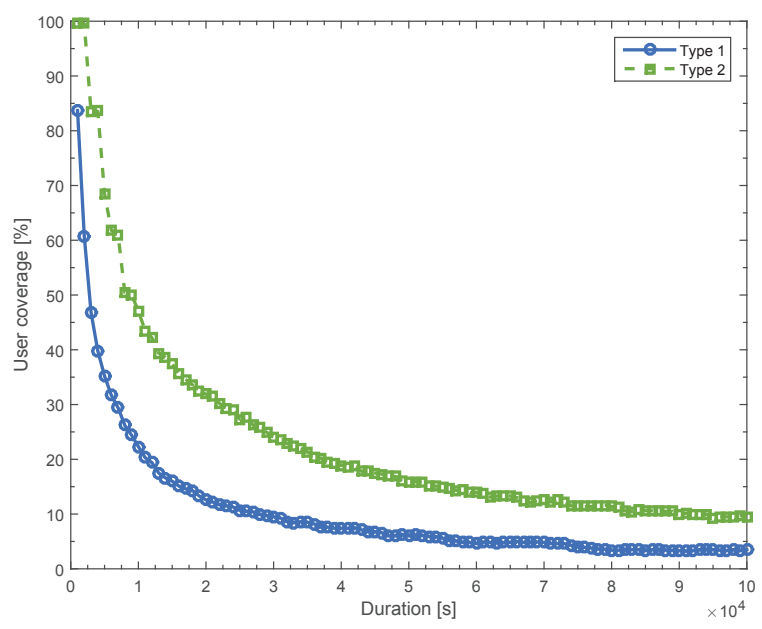

Fig. 6. Influence of the intervention duration on the user coverage.

Besides the influence on the user coverage, it is also interesting how many extra drones (besides the 300 drones already available as mentioned above) will be needed to cover all users when varying the intervention duration. As presented in Fig. 7, this relation is linear. When the intervention duration doubles, the number of extra drones needed also doubles (825 drones extra for 4000s versus 1859 for 8000s). This is logical as the longer the intervention lasts, the more drones will be needed to cover the users continuously. It is evident that the amount of drones needed is proportionally with the intervention duration. The same trend is noticed for the Type 2 drones, however, due to their longer fly time the total amount of additional drones is lower.

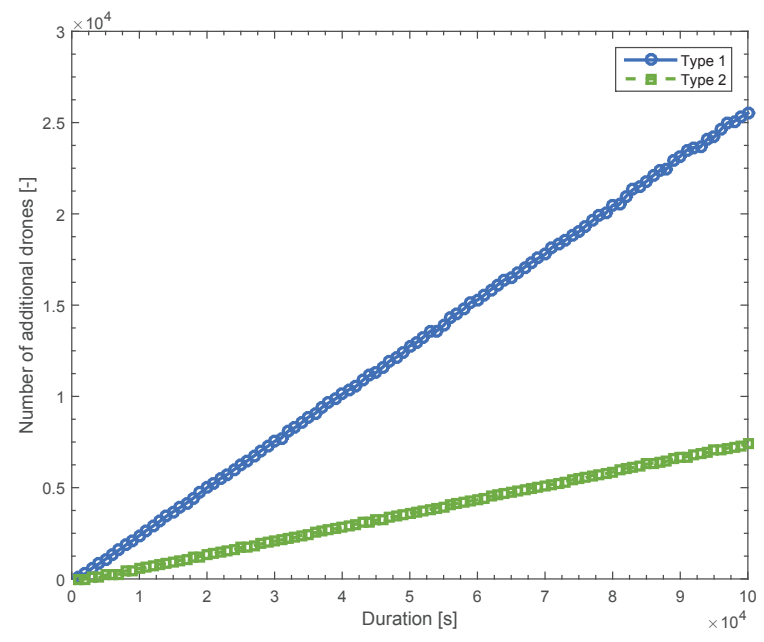

Fig. 7. Number of extra drones needed to cover all users as a function of the intervention duration. 


\section{Influence of the fly height of the drone}

The fly height of the drone will have its influence on the coverage of the base station carried by this drone and will thus have an influence on the user coverage and the amount of drones needed to cover all users. A fly height of $4 \mathrm{~m}$ means that the drone will fly $4 \mathrm{~m}$ above street level or above the building rooftop when located above a building as discussed in Section II-B. Fig. 8 visualizes the influence of the fly height on the user coverage. No maximal fly height $h_{\max }$ was set for these simulations in order to investigate the full impact of the fly height.

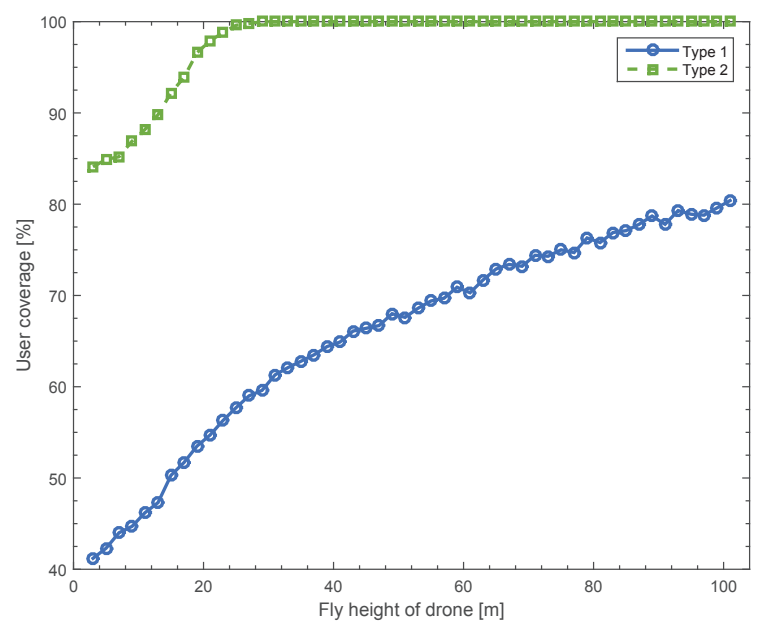

Fig. 8. Influence of the drone's fly height on the user coverage.

Fig. 8 shows that a higher fly height corresponds with a higher user coverage. For example when we increase the fly height from $5 \mathrm{~m}$ to $15 \mathrm{~m}$ for the Type 1 drone, the user coverage increases with $19 \%$ (42.3\% versus $61.3 \%$ ). For the Type 2 drone, a user coverage of $99 \%$ is reached when using a fly height of $23 \mathrm{~m}$, and all users are covered when the drones fly on height equal or higher than $29 \mathrm{~m}$. The higher the drone flies, the higher the coverage of the base station mounted on this drone will be, and thus the more users can be covered. This is due to the fact that there will be less obstructions and the users will be more in Line-of-Sight of the base stations mounted on the drones. As a higher flying drone results in a higher user coverage, one can expect that less drones will be needed to have full user coverage when we allow them a higher fly height. Fig. 9 shows this relation.

The higher the drones fly, the lower the number of drones needed to cover all users. Tripling the fly height from $5 \mathrm{~m}$ to $15 \mathrm{~m}$ reduces the number of needed drones by $21.9 \%$ (969 versus 757) for the Type 1 drone. When using the Type 2 drone, 283 drones are needed at a fly height of $29 \mathrm{~m}$ to cover all users. If we double this fly height to $59 \mathrm{~m}$, the number of needed drones decreases with $23 \%$ to 218 drones in total. The same reason as mentioned above applies here: the higher the drone flies, the higher the coverage of the base station mounted on this drone and the lower the number of drones needed to

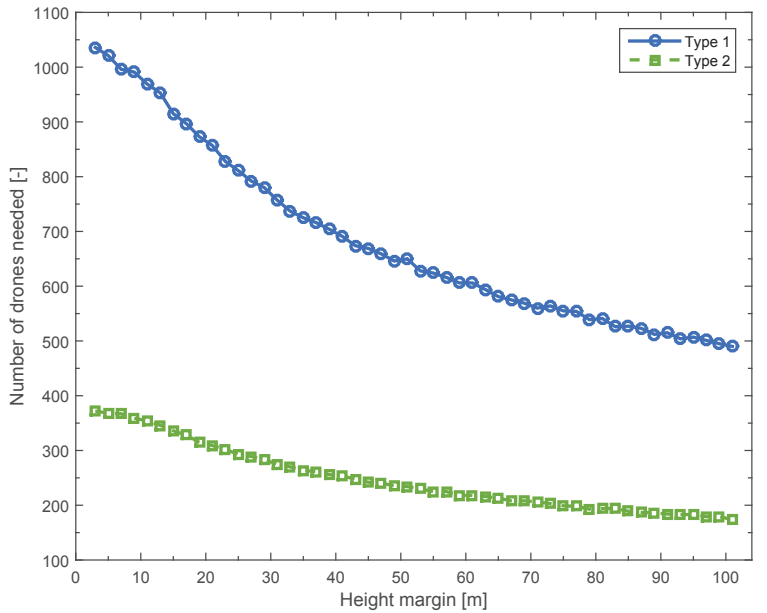

Fig. 9. Influence of the drone's fly height on the number of drones needed to cover all users.

have a $100 \%$ user coverage. However, there will be an upper limit for the user coverage and a lower limit for the number of drones. Both Figs. 8 and 9 indicate the evolution towards this limit: a higher fly height of the drone also results in a higher power usage. Increasing the fly height by $15 \mathrm{~m}$ results in an additional power usage of $0.5 \mathrm{~A}$. The drone will need additional power to fly further to bridge the height difference. In some cases the base station will also require extra power due to the higher input power to expand its coverage even further.

\section{CONCLUSION}

Today's wireless access networks are very reliable. However, on some rare occasions, the network fails to provide the omnipresent connectivity we are accustomed to. Unfortunately, the network becomes saturated when we actually need this connectivity the most, like for example during a natural disaster or a terrorist attack. In this study, the potential of mounting LTE femtocell base stations on drones, to offer a solution for those scenario where the fixed infrastructure is overwhelmed, is investigated. To this end, a deployment tool was developed which allows to determine not only the needed amount of drones, but also the most optimal locations of these drones in order to maximize the user coverage. The preliminary results of this study show that it is promising to investigate the use of drones for emergency scenarios. Based on the considered scenario and assumptions for the drone, approximately 1100 drones where needed to cover all users in the city center of Ghent, Belgium during $1 \mathrm{~h}$, when using an off the shelf drone that is affordable for the big public. When using a more advanced and powerful drone, only 400 drones where needed. The number of required drones can be significantly reduced by decreasing the user coverage requirement. Furthermore, the influence of the intervention duration and the fly height of the drones on the results is studied. The number of required drones can be reduced by $22 \%$ when tripling, respectively 
doubling, the fly height of the first, respectively second type, of drone. Our results clearly show that more research is needed including other types of drones, different bit rate requirements (e.g., only sending text messages), cost calculations, and also the connection with the back haul network, which was beyond the scope of this paper.

\section{ACKNOWLEDGMENT}

Margot Deruyck is supported by a postdoctoral grant from the Special Research Fund (BOF) of Ghent University (Belgium).

\section{REFERENCES}

[1] The Google Loon project, http://www.google.com/loon, Accessed: 19-042016.

[2] I. Bekmezci, O. K. Sahingoz, A. Temel, Flying Ad-Hoc Networks (FANETs): A survey, Ad Hoc Networks, Vol. 11, No. 3, 2013, pp. 12541270.

[3] M. Bennis, M. Simsek, A. Czylwik, W. Saad, S. Valentin, M. Debbah, When cellular meets WiFi in wireless small cell networks, IEEE Communications Magazine, Vol. 51, No. 6, 2013, pp. 44-50.

[4] M. Deruyck, D. De Vulder, W. Joseph, L. Martens, Modelling the Power Consumption in Femtocell Networks, IEEE Wireless Communications and Networking Conference, 2012, pp. 30-35.

[5] M. Deruyck, W. Joseph, E. Tanghe, L. Martens, Reducing the Power Consumption in LTE-Advanced Wireless Access Networks by a Capacity Based Deployment Tool, Radio Science, Vol. 49, No. 9, 2014, pp. 777787.

[6] COST telecommunications, COST Action 231: Digital Mobile Radio towards Future Generation Systems, European Commission, 1999.

[7] T. Reed, J. Geis, S. Dietrich, SkyNET: a 3G-enabled mobile attack drone and stealth botmaster Approach Attack Framework, 5th USENIX Conference on Offensive Technologies (WOOT), 2011, pp. 4.

[8] J. Valente, D. Sanz, A. Barrientos, J. Del Cerro, A. Ribeiro, C. Rossi, An Air-Ground Wireless Sensor Network for Crop Monitoring, Sensors, Vol. 11, No. 12, 2011, pp. 6088-6108.

[9] H. T. Kung, C.-K. Lin, T.-H. Lin, S.J. Tarsa, D. Vlah, Measuring diversity on a low-altitude UAV in a ground-to-air wireless 802.11 mesh network, GLOBECOM Workshops, 2010, pp. 1799-1804.

[10] W. Joseph, S. Aerts, M. Vandenbossche, A. Thielens, L. Martens, Drone Based Measurement System for Radiofrequency Exposure Assessment, Bioelectromagnetics, Vol. 37, No. 3, 2016, pp. 195-199.

[11] Microdrones MD4-1000: Robust and powerful UAV, https://www. microdrones.com/en/products/md4-1000/technical-data/, Accessed: 201606-20. 